\title{
A New Call Admission Control Strategy for LTE Femtocell Networks
}

\author{
Jie Wang, Yangfan Qiu \\ National Mobile Communication Research Lab (NCRL), Southeast University \\ Nanjing, 210096, China \\ Email: wangjie@seu.edu.cn
}

\begin{abstract}
This paper introduces a call admission control (CAC) strategy for LTE femtocell networks supporting multimedia services with different classes of traffic and diverse bandwidth requirements. In this work, the multiservice CAC strategy composes of two parts: subscriber authentication and queuing admission control. Theoretical analysis and simulation are performed on three key principles. Comparing with the typical strategy, simulation results indicate that better probabilistic connection-level QoS parameters are achieved by using the proposed strategy.
\end{abstract}

Keywords-femtocell; HeNB; LTE; CAC; CSG

\section{INTRODUCTION}

Femtocell is a low-cost and low-power cellular home base station that works on the licensed spectrum and connects mobile users to the operator's core network over the Internet via broadband backhaul. With the help of femtocell technology, the next generation mobile communication systems can provide faster and more reliable indoor communication [1]. As a result, there is a growing interest in deploying multimedia services in indoor mobile cellular networks. However, several aspects related to the macro/femto-cell hierarchical structure are still remaining to be adapted. Call admission control (CAC) is one of such areas in need of adaptation to accommodate multimedia services.

Typical CAC schemes of multi-service are complete sharing (CS), complete partitioning (CP) and threshold [2]. In the CS scheme, calls of every class of services share the bandwidth pool [3]. Whereas, in the CP scheme, the bandwidth for each class of services is exclusively reserved [4]. In the threshold scheme, a newly arriving call is blocked if the number of calls of corresponding service is greater than or equal to a predefined threshold. It is proven that the threshold scheme is optimal in two-class network [5]. However, the threshold scheme is not optimal in general multi-service networks.

There are three access modes which allow users to access femtocell: close access, open access, hybrid access. The close access mode only allows the closed subscriber group (CSG) members entering the CSG's femtocell, the open access mode enables all users to share the resource provided by the femtocell. The hybrid access mode is a combination of both, it affords other UEs to access the CSG's femtocell. Normally, the hybrid access femto BS is owned by CSG member subscribers, there is discrimination for service between CSG members and non-CSG members. Service quality for CSG members should not be affected by non-CSG members. Therefore it has become necessary that CAC considering priority control for CSG members.

Many works have been conducted in the area of the CAC strategy in hierarchical macro/femto-cell networks, but most of them focus on the policies for different access modes. In [6], the authors considered the different admission control policies for femtocell operation, proposed an operating region where femto BS has motivation to operate in open access mode. An optimization CAC mechanism in hybrid access mode based on the discrimination for service between CSG members and non-CSG members was described in [7]. Reference [8] studied the femtocell access control strategy for UMTS and LTE was provided based on 3GPP Release 8 specifications. In this paper, we consider multi-service call admission control for LTE femtocell networks.

The rest of the paper is organized as follows. Next section describes the proposed call admission control strategy. The simulation scenario and parameters are explained in Section III. Numerical results are discussed in Section IV and the paper is concluded in Section V.

\section{Proposed CAll AdMission CONTROL STRATEGY}

A bearer is an IP packet flow with a defined Quality of Service (QoS). E-UTRAN Radio Access Bearer (E-RAB) refers to the concatenation of an S1 bearer and the corresponding radio bearer. LTE uses the concept of ERAB to route the packets of an Evolved Packet Core (EPS) bearer between a serving gateway (S-GW) and a user equipment (UE) [9]. The task of call admission control in LTE is to decide whether or not to establish new E-RAB.

With the three access modes we mentioned before, the femto BS is a private station, therefore the CAC strategy for femtocell should compose of two parts: subscriber authentication and admission control. The flowchart in Fig. 1 describes the proposed CAC process. 


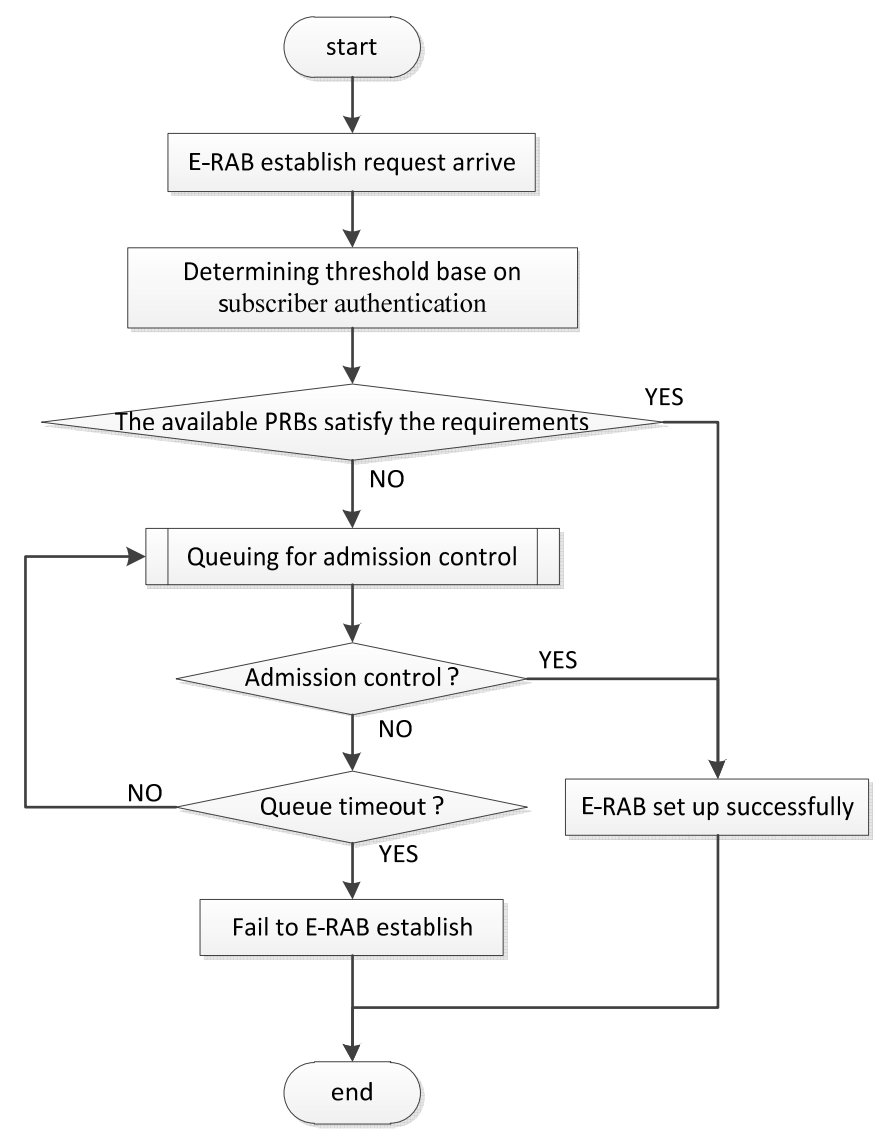

Figure 1. Femtocell call admission control process

In the following section, we describe three key principles of the proposed call admission control strategy.

\section{A. Threshold base on subscriber authentication}

Generally, admission control algorithm estimates the demand for physical resource block (PRB) $P R B_{i}^{\text {demand }}$ of service $i$, when a bear establish request arrives. We assume base station has $P R B_{i}^{\text {total }}$ amount of PRBs can be allocated to service class $i$. With the occupied PRBs $P R B_{i}^{\text {used }}$ and the remainder $\operatorname{PRBs} P R B_{i}^{\text {avail }}$, there are following relationships:

$$
\begin{gathered}
P R B_{i}^{\text {avail }}=P R B_{i}^{\text {total }}-P R B_{i}^{\text {used }} \\
P R B_{i}^{\text {used }}=\sum_{u=1}^{k} \frac{R_{i}^{u}}{T B_{u}^{\text {mcs }}}
\end{gathered}
$$

Here $R_{i}^{u}$ and $T B_{u}^{m c s}$ represent the rate of user $u$ and the amount of transport block (TB) with current modulation and coding scheme (MCS).

The criterion for admission can be expressed as follow:

$$
P R B_{i}^{\text {demand }} \leq \delta P R B_{i}^{\text {avail }}
$$

$\delta$ is the threshold decided during subscriber authentication which has different policies for CSG members with high priority. As a result, $\delta$ of CSG members $\delta_{C S G}$ and $\delta$ of non-CSG members $\delta_{\text {non-CSG }}$ have following relationship:

$$
\delta_{\text {CSG }}>\delta_{\text {non-CSG }}
$$

\section{B. Queuing priority base on multimedia service}

For the sake of clarity, multimedia services belong to multiple types will be referred to three classes: conversational class, streaming class, best effort class. Conversational class service represents real-time traffic such as VoIP, therefore we focus on its handoff call dropping probability and allocate guard channel (GC) for the handoff calls. Streaming class service is another kind of real-time traffic such as online video, but thanks to the video buffer it is not as delay-sensitive as conversational class service. Best effort class service represents non-realtime traffic such as FTP, it is more sensitive to error rate, and has low requirement for delay.

Our queuing model is shown in Fig. 2. In addition to the GC for the conversational class service, the resource of system is divided into two parts. One of them is the special resource for best effort class service, the other one is the public resource which the real-time traffic can perform preemption in case of radio congestion.

We take the GC as a dynamic proportion rather than fixed amount of PRBs. Newly arriving conversational calls are blocked if the amount of available PRBs are less than the dynamic proportion of total PRBs, however the conversational handoff calls are blocked only when all PRBs are exhausted.

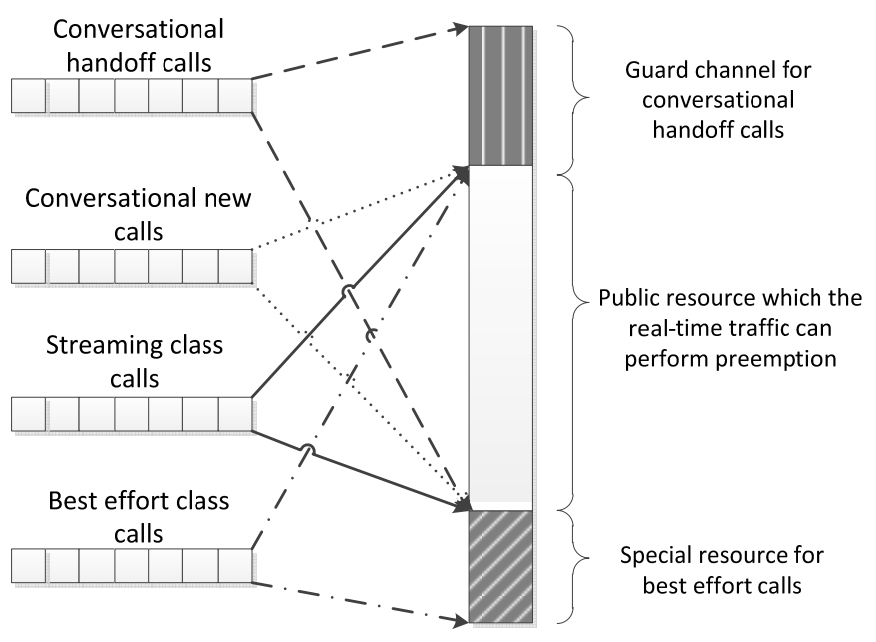

Figure 2. Queuing model for multimedia service

Note that a service is associated with a queue, and the queue length needs to be monitored. Once the length of queue exceeds a certain size, the next coming calls should be discarded. Moreover, there are corresponding timers for each queue; the call waiting in the queue would be discarded when a timeout occurs. 


\section{Dynamic GC base on reinforcement learning}

GC makes handoff calls have higher priority, so that the handoff calls can get expected QoS requirement. However, allocating GC will certainly raise new call blocking probability. We model the update process of the dynamic GC as a Markov decision process (MDP), which consists of a set of states and actions aiming at finding a policy that minimizes the corresponding cost for each state [10].

In particular, at time $t$ the system state is defined as $s_{t}=\left\{G, L_{\text {con }}^{\text {ho }}, L_{\text {con }}^{\text {new }}\right\} . G \in\left\{g_{1}, g_{2}, \ldots, g_{l}\right\}, g_{i}<g_{j}, i<j$ is the set of $l$ level proportion, which GC can set to. $L_{c o n}^{\text {ho }}, L_{c o n}^{\text {new }}$ are the queue length of conversational handoff calls and the queue length of conversational new calls. Let action $a_{t} \in\left\{a^{1}, a^{2}, a^{3}\right\}$, here $a^{1}$ and $a^{2}$ mean to degrade the state and upgrade the proportion level, while $a^{3}$ means to maintain the current proportion. After observing the current state, an action has to be made and the corresponding cost $c\left(s_{t}, a_{t}\right)$ as well as the new state is gained.

We set the cost as the following relationship:

$$
c=\omega_{\text {ho }} P_{h o}+\omega_{n} P_{n}
$$

Where $P_{h o}$ and $P_{n}$ represent the handoff call dropping probability and new call blocking probability respectively, $\omega_{h o}$ and $\omega_{n}$ are their weights. As it should pay more attention to handoff call dropping probability, $\omega_{\text {ho }}$ must larger than $\omega_{n}$,

With the learning procedure and more specifically the application of Q-learning model, our objective is to find an optimal policy $\pi^{*}\left(s_{t}\right)$ for each state to minimize cumulative measure of the cost.

The expected discounted cost over an infinite horizon is given by:

$$
\begin{aligned}
V^{\pi}(s)= & E\left\{\sum_{t=0}^{\infty} \Upsilon^{t} c\left(s_{t}, \pi\left(s_{t}\right)\right) \mid s_{0}=s\right\} \\
= & E\left\{c\left(s_{0}, a_{0}\right) \mid s_{0}=s, a_{0}=a\right\}+ \\
& E\left\{\sum_{t=1}^{\infty} \Upsilon^{t} c\left(s_{t}, \pi\left(s_{t}\right)\right) \mid s_{0}=s, a_{0}=a\right\} \\
= & E\{c(s, a)\}+ \\
& \Upsilon \sum_{s^{\prime} \in S} P_{s, s^{\prime}}(a) E\left\{\sum_{t=1}^{\infty} \Upsilon^{t-1} c\left(s_{t}, \pi\left(s_{t}\right)\right) \mid s_{1}=s^{\prime}\right\} \\
= & C(s, a)+\Upsilon \sum_{s^{\prime} \in S} P_{s, s^{\prime}}(a) V^{\pi}\left(s^{\prime}\right)
\end{aligned}
$$

Where $C(s, a)=E\{c(s, a)\}, \Upsilon \in(0,1)$ is a discount factor. Equation (7) denotes that the Q-function of the current state-action pair, Q-learning process finding $\pi^{*}\left(s_{t}\right)$ in a recursive manner which is given in Eq. (8) and Eq. (9), here $\alpha \in(0,1)$ is a learning rate [11].

$$
\begin{gathered}
Q(s, a)=\mathrm{C}(s, a)+\Upsilon \sum_{s^{\prime} \in S} P_{s, s^{\prime}}(a) V^{\pi}\left(s^{\prime}\right) \\
Q\left(s_{t}, a_{t}\right)=(1-\alpha) Q\left(s_{t}, a_{t}\right)+\alpha\left[c\left(s_{t}, a_{t}\right)+\Upsilon \min _{a_{t+1}} Q\left(s_{t+1}, a_{t+1}\right)\right] \\
\pi^{*}\left(s_{t}\right)=\min _{\pi} V^{\pi}\left(s_{t}\right) \\
=\min _{\pi} E\left\{\sum_{t=0}^{\infty} \Upsilon^{t} c\left(s_{t}, \pi\left(s_{t}\right)\right) \mid s_{0}=s\right\} \\
=\min _{\pi} Q\left(s_{t}, a_{t}\right)
\end{gathered}
$$

\section{SimUlation SCENARIO AND PARAMETERS}

We consider a single floor building with 25 apartments. The apartments are of size $10 \mathrm{~m} \times 10 \mathrm{~m}$ and are placed next to each other on a $5 \times 5$ grid as shown in Fig 3 [12]. There is a femtocell in each apartment, the femto BS resides at the center of each femtocell. UEs are dropped randomly and uniformly in the apartment. The dwell time that a UE stays in a femtocell before handoff is assumed to follow an exponential distribution with mean $1 / \eta$. All the services follow the same dwell time distribution. Note that the parameter $\eta$ represents the handoff rate; moreover the UE is assumed to handoff to its adjacent femtocells with equal probability.

Services are generated according to a Poisson process with rate $\lambda$ (calls/second). The life time of the service $i$ is exponentially distributed with mean $1 / \mu_{i}$. Traffic intensity denotes as $\rho=\lambda_{i} / \mu_{i}$. For brevity, we consider three services: VoIP, video stream, CBR. We set the ratio

\begin{tabular}{|c|c|}
\hline Parameter & Values \\
\hline Carrier frequency & $2 \mathrm{GHz}$ \\
\hline Signal bandwidth & 5MHz (25 PRBs) \\
\hline Tx power of femto cell & $20 \mathrm{dBm}$ \\
\hline Height of femto cell & $3 m$ \\
\hline Wall loss (WL) & $10 \mathrm{~dB}$ \\
\hline Pathloss (femto) & $\begin{array}{c}\max \left\{\begin{array}{l}15.3+37.6 \times \log _{10}(d[\mathrm{~m}]), \\
37+20 \times \log _{10}(d[\mathrm{~m}])\end{array}\right\}+W L ; \\
37+20 \times \log _{10}(d[\mathrm{~m}])+W L \\
\text { (outdoor/indoor) }\end{array}$ \\
\hline
\end{tabular}
of call arrive rate of as 5:2:3, that is $\lambda_{\text {voip }}=0.5 \lambda, \lambda_{\text {vedio }}=0.2 \lambda, \lambda_{\text {cbr }}=0.3 \lambda$ [13]. All the services are modeled with an ON/OFF Markov chain. During the ON period, the voice flow sends 30 bytes sized packets every 20ms [14], the video stream sends packets based on realistic video trace files which are available on [15]. CBR application represents the best effort service which generates packets with a constant bit rate 64 kbps [16]. The size of all queues is fixed to be 5 . The other simulation parameters are given in Table I.

TABLE I. SIMULATION PARAMETERS 


\begin{tabular}{|l|c|}
\hline Log-normal shadowing & Standard deviation $=4 \mathrm{~dB}$ \\
\hline Auto-correlation distance & $3 \mathrm{~m}$ \\
\hline Threshold & $\delta_{C S G}=1, \delta_{\text {non-CSG }}=0.8$ \\
\hline Weights & $\omega_{\text {ho }}=0.8 . \omega_{n}=0.2$ \\
\hline
\end{tabular}

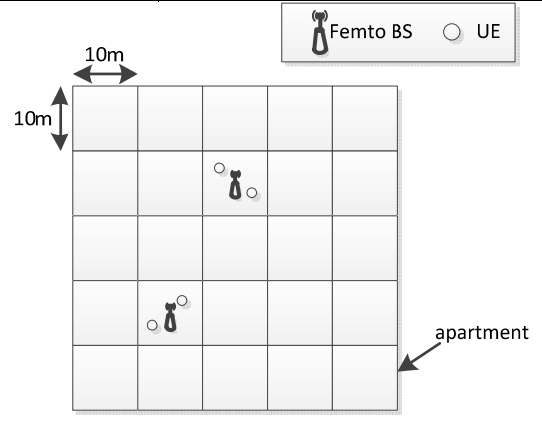

Figure 3. $5 \times 5$-grid model

\section{Simulation Results}

In first set of simulations, we compare the performance of complete sharing scheme, complete partitioning scheme and proposed queuing priority scheme. The performance can be quantitatively expressed in terms of probabilistic connection-level QoS parameters such as call blocking probability. As expected, CS can maintain high level quality of high priority service such as VoIP, at the expense of decreasing performance of low priority service. However, CP can ensure the performance of each class of services, but the resource utilization is relatively low. Numerical results show that the service of both high priority traffic and low priority traffic is guaranteed by the proposed scheme, and moreover, the resource utilization is promoted.

Due to the limited space we just show a part of simulation results. As shown in Fig. 4, the blocking probability of VoIP increases with the call arrive rate increases. However, the proposed queue priority scheme provides significant reduction in blocking probability compared to the complete partitioning scheme. Figure 5 illustrates that the proposed scheme make the PRB utilization of CBR as high as complete partitioning scheme.

Figure 6 shows the average cost as a function of handoff rate $\eta$. The cost is calculated as Eq. (5) while VoIP accounts for 50 percent of the overall traffic. It can be observed that as $\eta$ increases the cost of rigid divisionbased GC increases, however, proposed dynamic GC maintain the cost at a low level.

\section{CONCLUSION}

In this paper, a multi-service call admission control strategy for LTE femtocell networks is proposed. The proposed strategy considers multiple classes of services with different QoS requirement. Three related components comprise the main building block of the strategy: (1) threshold base on subscriber authentication, (2) Queuing priority base on multimedia service, (3) Dynamic guard channel base on reinforcement learning. Simulation results demonstrate that our proposed strategy can guarantee the call blocking probability for each class of traffic, while the resource utilization is also promoted. It is also shown the proposed dynamic guard channel scheme is a solution to the contradiction between call handover dropping and congestion of new calls.

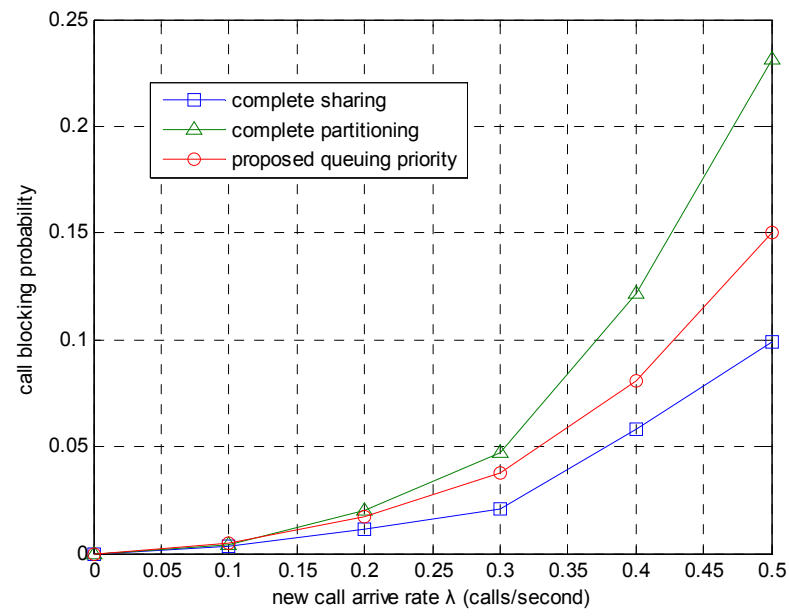

Figure 4. Call blocking probability of VoIP

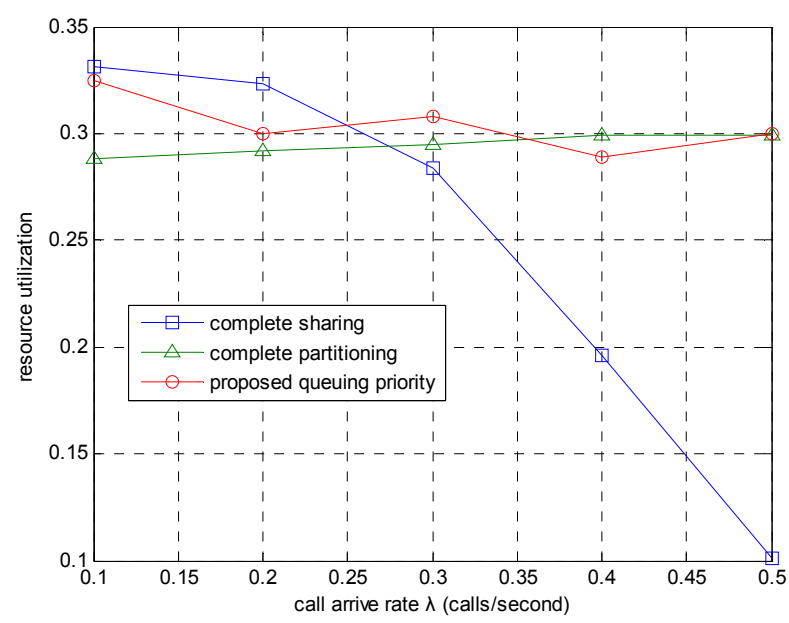

Figure 5. Resource utilization of CBR 


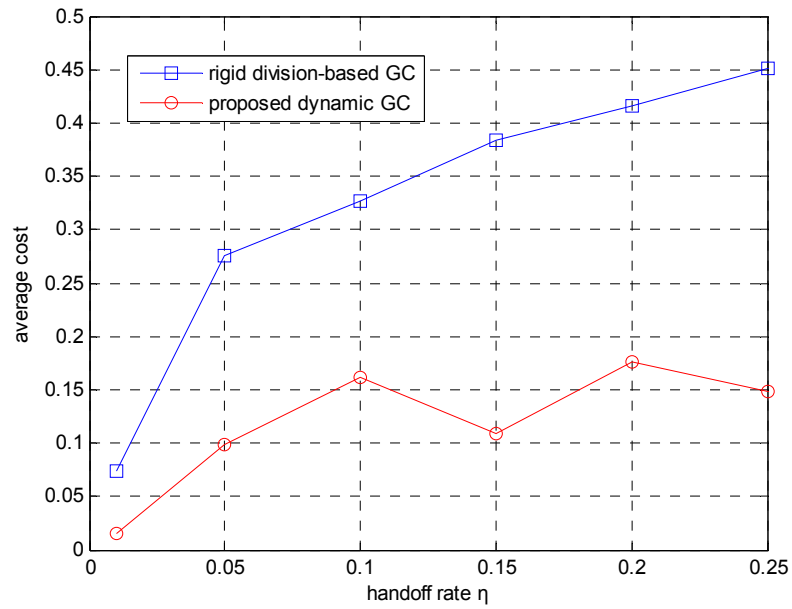

Figure 6. Average cost as a funciton of hanoff rate $\eta$

\section{REFERENCES}

[1] V. Chandrasekhar, J. G. Andrews, and A. Gatherer, "Femtocell networks: a survey,” IEEE Communications Magazeine, vol.46, no. 9, pp. 59-67, Sept. 2008.

[2] Jihyuk Choi, Taekyoung Kwon, Yanghee Choi, Naghshineh M, "Call admission control for multimedia services in mobile cellular networks: a Markov decision approach,” ISCC 2000, July 2000, pp.594-599.

[3] R.A.Guerin, "Queuing blocking system with two arrival atreams and guard channels,” IEEE Transactions on Commnication Vol. 36, No. 2, pp.153-163, Feb. 1988
[4] S. A. AlQahtani, Hassan Mahmoud and A. Alshanyour, "Performance evaluation and analytical modeling of nove dynamic call admission control scheme for 3G and beyond celluar wireless networks,” AICCSA 2007, May 2007, pp. 631-638.

[5] Keith W. Ross and Danny H. K. Tsang, "The stochastic knapsack problem,” IEEE Transactions on Communications, Vol. 37, No. 7, pp.740-747, July 1989.

[6] M. E. R. Khan, "Fair admission control policies for femtocel operation,” ICUFN 2012, July 2012, pp. 175-179.

[7] Seung Que LEE, RYU Bying Han and Nam-Hoon PARK, "Call admission control for hybrid access mode femtocell system,” WiMob 2011, Oct. 2011, pp. 512-516.

[8] A. Golaup, M. Mustapha and L. B. Patanapongpobul, "Femtocell access control strategy in UMTS and LTE,” IEEE Commun. Mag. , Vol. 47, No. 9, pp. 117-123, Sept. 2009.

[9] Stefania sesia, Issam Toufik, Matthew Baker, LTE-The UMTS Long Term Evolution: From Theory to Practice, Wiley, 2011.

[10] M. E. Harmon and S. S. Harmon, "Reinforcement learning: A tutorial," 2000.

[11] C. J. Watkins and P. Dayan, “Technical note: Q-learning," Machine Learning, Vol. 8, pp. 279-292, 1992.

[12] M. Simsek et al, "An LTE-femtocell dynamic system level simulator,” in proc. IEEE smart antennas (WSA), 2010 international ITG Workshop, pp. 66-71, Feb. 2010.

[13] IEEE 802.16m Evaluation Methodology Document (EMD).

[14] Giuseppe Piro et al., "Simulating LTE cellular systems: an open source Framework,” IEEE Trans. Veh. Technol., Vol. 60, No. 2, pp. 498-513, Oct. 2010

[15] “Video trace library,” [OnLine] Available: http://trace.eas.asu.edu/.

[16] Hjelm.B, "admission control in future multi-service wideband direct-sequence CDMA systems,” IEEE VTC-FALL 2000. Vol. 3, pp. 1086-1093, Sept. 2000. 